PROCEEDINGS OF THE

AMERICAN MATHEMATICAL SOCIETY

Volume 136, Number 11, November 2008, Pages 3769-3774

S 0002-9939(08)09465-3

Article electronically published on June 24, 2008

\title{
ON A DESINGULARIZATION OF THE MODULI SPACE OF NONCOMMUTATIVE TORI
}

\author{
IGOR NIKOLAEV \\ (Communicated by Marius Junge)
}

\begin{abstract}
It is shown that the moduli space of the noncommutative tori $\mathbb{A}_{\theta}$ admits a natural desingularization by the group $\operatorname{Ext}\left(\mathbb{A}_{\theta}, \mathbb{A}_{\theta}\right)$. Namely, we prove that the moduli space of pairs $\left(\mathbb{A}_{\theta}, \operatorname{Ext}\left(\mathbb{A}_{\theta}, \mathbb{A}_{\theta}\right)\right)$ is homeomorphic to a punctured two-dimensional sphere. The proof is based on a correspondence (a covariant functor) between the complex and noncommutative tori.
\end{abstract}

\section{INTRODUCTION}

A. Let $0<\theta<1$ be an irrational number, whose regular continued fraction has the form $\theta=\left[a_{0}, a_{1}, a_{2}, \ldots\right]$. Consider an $A F$-algebra $\mathbb{A}_{\theta}$ given by the Bratteli diagram in Figure 1. The $a_{i}$ indicate the number of edges in the upper row of

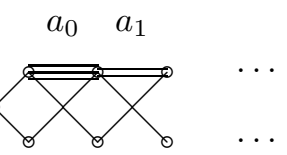

Figure 1. The Bratteli diagram of the $A F$-algebra $\mathbb{A}_{\theta}$.

the diagram. With a moderate abuse of the terminology, we shall call $\mathbb{A}_{\theta}$ a noncommutative torus. (Note that a standard definition of the noncommutative torus - a universal $C^{*}$-algebra generated by the unitaries $u, v$ satisfying the commutation relation $v u=e^{2 \pi i} u v$ - is not an $A F$-algebra. However, the two objects are isomorphic at the level of their dimension groups [8, 9].)

B. Recall that the noncommutative tori $\mathbb{A}_{\theta}, \mathbb{A}_{\theta^{\prime}}$ are said to be stably isomorphic, whenever $\mathbb{A}_{\theta} \otimes \mathcal{K} \cong \mathbb{A}_{\theta^{\prime}} \otimes \mathcal{K}$, where $\mathcal{K}$ is the $A F$-algebra of the compact operators. It is well known that the $A F$-algebras $\mathbb{A}_{\theta}, \mathbb{A}_{\theta^{\prime}}$ are stably isomorphic if and only if $\theta^{\prime} \equiv \theta \bmod S L(2, \mathbb{Z})$, i.e. $\theta^{\prime}=(a \theta+b) /(c \theta+d)$, where $a, b, c, d \in \mathbb{Z}$ and $a d-b c=1[3$. It is easy to see that the stable isomorphism is an equivalence relation, which splits the set $\left\{\mathbb{A}_{\theta} \mid 0<\theta<1, \theta \in \mathbb{R}-\mathbb{Q}\right\}$ into the disjoint equivalence classes. By $\mathcal{M}$ we shall understand a collection of such classes, or the

Received by the editors March 30, 2007, and, in revised form, September 6, 2007.

2000 Mathematics Subject Classification. Primary 14H52, 46L85.

Key words and phrases. Complex tori, noncommutative tori.

The author was partially supported by NSERC.

(C)2008 American Mathematical Society

Reverts to public domain 28 years from publication 
"moduli space" of the noncommutative tori. An examination of $\mathcal{M}$ as a topological space (with the topology induced by $\mathbb{R}$ ) shows that the points of $\mathcal{M}$ have no disjoint neighborhoods, since each orbit $\left\{\theta^{\prime} \in \mathbb{R} \mid \theta^{\prime} \equiv \theta \bmod S L(2, \mathbb{Z})\right\}$ is dense in the real line $\mathbb{R}$. A question arises as to how to "desingularize" the (non-Hausdorff) moduli space $\mathcal{M}$.

C. Let $A, B$ be a pair of the $C^{*}$-algebras. Recall that an extension of $A$ by $B$ is a $C^{*}$-algebra $E$ filling the short exact sequence $0 \rightarrow B \rightarrow E \rightarrow A \rightarrow 0$ of the $C^{*}$-algebras. If $A$ is a separable nuclear $C^{*}$-algebra, the $\operatorname{Ext}(A, B)$ is an additive abelian group, whose group operation is a sum of the two extensions. The $\operatorname{Ext}(A, B)$ is a homotopy invariant in both variables. The extensions $E_{1}, E_{2}$ are said to be stably equivalent if there exists an isomorphism $\psi: E_{1} \otimes \mathcal{K} \cong E_{2} \otimes \mathcal{K}$, such that $\psi \circ \alpha_{1}(B \otimes \mathcal{K})=\alpha_{2}(B \otimes \mathcal{K})$, where $\alpha_{i}: B \rightarrow E_{i}, i=1,2$ [1]. We shall further restrict to the case $A=B=\mathbb{A}_{\theta}$ and study the stable equivalence classes of the group Ext $\left(\mathbb{A}_{\theta}, \mathbb{A}_{\theta}\right)$. Using the classification results of D. Handelman [5], it will develop that the group Ext $\left(\mathbb{A}_{\theta}, \mathbb{A}_{\theta}\right) \cong \operatorname{Hom}\left(K_{0}\left(\mathbb{A}_{\theta}\right), \mathbb{R}\right) \cong \mathbb{R}$. Moreover, the $\operatorname{Ext}\left(\mathbb{A}_{\theta}, \mathbb{A}_{\theta}\right) /$ stable equivalence $\cong \mathbb{R} / \mathbb{Z}$.

D. An objective of the note is to show that the moduli of the pairs $\left(\mathbb{A}_{\theta}, \operatorname{Ext}\left(\mathbb{A}_{\theta}\right.\right.$, $\left.\mathbb{A}_{\theta}\right)$ ) under the stable equivalence is no longer a non-Hausdorff topological space, but a two-dimensional orbifold (a punctured sphere). To prove this result we shall use the Teichmüller space of a torus (a space of the complex structures on the torus) 6]. Namely, Hubbard and Masur established a homeomorphism between the Teichmüller space $T_{g}$ of a surface of genus $g \geq 1$ and the space of quadratic differentials on it. We shall use the homeomorphism to extend the action of the modular group $S L(2, \mathbb{Z})$ from the upper half-plane $\mathbb{H}=\{x+i y \in \mathbb{C} \mid y>0\} \cong T_{1}$ to the space $\left(\mathbb{A}_{\theta}, \operatorname{Ext}\left(\mathbb{A}_{\theta}, \mathbb{A}_{\theta}\right)\right)$. Denote by $\widetilde{\mathcal{M}}$ the set of pairs $\left(\mathbb{A}_{\theta}, \operatorname{Ext}\left(\mathbb{A}_{\theta}, \mathbb{A}_{\theta}\right)\right)$ modulo the stable equivalence. One obtains the following (natural) desingularization of the moduli space of the noncommutative tori.

Theorem 1. $\widetilde{\mathcal{M}}$ is a punctured two-dimensional sphere.

\section{Proof}

We shall split the proof into two lemmas. The background material is mostly standard, and we shall recall in passing some important notation and ideas.

Lemma 1. $\widetilde{\mathcal{M}}$ is a two-dimensional orbifold.

Proof of Lemma 1. We shall use a standard dictionary existing between the $A F$ algebras and their dimension groups 2 . Instead of dealing with the $A F$-algebra $\mathbb{A}_{\theta}$, we shall work with its dimension group $G_{\theta}=\left(G, G^{+}\right)$, where $G \cong \mathbb{Z}^{2}$ is the lattice and $G^{+}=\left\{(x, y) \in \mathbb{Z}^{2} \mid x+\theta y \geq 0\right\}$ is a positive cone of the lattice. The $G_{\theta}$ is the additive abelian group with an order, which defines the $A F$-algebra $\mathbb{A}_{\theta}$ up to a stable isomorphism.

Under the dictionary, the extension problem for the $A F$-algebra $\mathbb{A}_{\theta}$ translates as an extension problem for the dimension groups $G_{\theta} \rightarrow E \rightarrow G_{\theta}$ (we omit the zeros in the exact sequence). An important result of Handelman establishes the intrinsic classification of the extensions of the simple dimension group by a simple dimension group; see Theorem III.5 of [5]. Let us recall the classification as it is exposed in [4. Theorem 17.5 and Corollary 17.7]. We shall adopt the same notation as in the cited work. 
Let $H$ be a dense subgroup of the real line $\mathbb{R}$ and $K$ a nonzero dimension group. Let $E$ be the abelian group $H \oplus K$, and let $\tau: H \rightarrow E$ and $\pi: E \rightarrow K$ be a natural injection and projection maps. Assume that $f: K \rightarrow \mathbb{R}$ is a homomorphism of the dimension groups 1 Then: (i) $E$ is a dimension group with the positive cone

$$
E_{f}^{+}=\{(0,0)\} \cup\{(x, y) \in E \mid y \geq 0 \text { and } x+f(y)>0\},
$$

which gives an extension $H \stackrel{\tau}{\rightarrow}\left(E, E_{f}^{+}\right) \stackrel{\pi}{\rightarrow} K$ of $H$ by $K$; (ii) if $f, f^{\prime}: K \rightarrow \mathbb{R}$ are the group homomorphisms, then the extensions $E_{f}, E_{f^{\prime}}$ are equivalent if and only if $\left(f-f^{\prime}\right)(K) \subseteq H$.

We have to specialize the above theorem to the case $H=K=G_{\theta}$. It is immediate from (i) that $E \cong \mathbb{Z}^{4}$. Note that the group homomorphisms $f: G_{\theta} \rightarrow \mathbb{R}$ are bijective with the reals $\mathbb{R}$. Indeed, we have to find all the linear maps $f$ : $\mathbb{R}^{2} \rightarrow \mathbb{R}$, such that $\operatorname{Ker} f=x+\theta y$. (The last equation follows from the condition $f\left(G^{+}\right)>0$.) Such maps have the form $f_{t}(p)=(p, t), p, t \in \mathbb{R}^{2}$, where $(p, t)$ is the dot product of the two vectors. Let $t=\left(t_{1}, t_{2}\right)$. Then $f_{t}(-\theta y, y)=t_{1}(-\theta y)+t_{2} y=$ $y\left(t_{2}-t_{1} \theta\right)=0$ for all $y \in \mathbb{R}$. Therefore, $t_{2}=\theta t_{1}$ and $f_{t}(x, y)=t_{1} x+\theta t_{1} y=$ $t_{1}(x+\theta y), t_{1} \in \mathbb{R}$. Thus, all linear maps $f: \mathbb{R}^{2} \rightarrow \mathbb{R}$ with $\operatorname{Ker} f=x+\theta y$ are bijective with the reals $t_{1} \in \mathbb{R}$. In other words, Ext $\left(\mathbb{A}_{\theta}, \mathbb{A}_{\theta}\right)$ and $\mathbb{R}$ are isomorphic as additive abelian groups.

Let us find when the two extensions $E, E^{\prime}$ are equivalent. Since $H=G_{\theta}$ is a subgroup of $\mathbb{R}$, one can write $H=\mathbb{Z}+\theta \mathbb{Z}$. Let $t, t^{\prime}$ be the real numbers corresponding to the homomorphisms $f, f^{\prime}$. Then $f\left(G_{\theta}\right)=t(\mathbb{Z}+\theta \mathbb{Z})$ and $f^{\prime}\left(G_{\theta}\right)=t^{\prime}(\mathbb{Z}+\theta \mathbb{Z})$. The condition $\left(f-f^{\prime}\right)(K) \subseteq H$ of the item (ii) will take the form $\left(t-t^{\prime}\right)(\mathbb{Z}+\theta \mathbb{Z}) \subseteq \mathbb{Z}+\theta \mathbb{Z}$. One gets immediately that $t=t^{\prime}+n, n \in \mathbb{Z}$ as a necessary and sufficient condition for the last inclusion. In other words, the extensions $E, E^{\prime}$ are equivalent if and only if $t^{\prime}=t \bmod \mathbb{Z}$. Thus, the equivalence classes of $\operatorname{Ext}\left(\mathbb{A}_{\theta}, \mathbb{A}_{\theta}\right)$ are bijective with the factor space $\mathbb{R} / \mathbb{Z}$ (a unit interval).

To finish the proof of Lemma 1 let us extend the domain of definition of $\theta$ from the interval $(0,1)$ to the real line $\mathbb{R}$ by allowing $a_{0}$ to take on any integer value. In this way, one can identify the pairs $\left(\mathbb{A}_{\theta}, \operatorname{Ext}\left(\mathbb{A}_{\theta}, \mathbb{A}_{\theta}\right)\right)$ with the points of $\mathbb{R}^{2}$ equipped with the usual Euclidean topology. We have seen that the points $(\theta, t) \sim\left(\theta^{\prime}, t^{\prime}\right) \in \mathbb{R}^{2}$ are equivalent if and only if $\theta^{\prime} \equiv \theta \bmod S L(2, \mathbb{Z})$ and $t^{\prime} \equiv$ $t \bmod \mathbb{Z}$. Note that the action of the modular group on the second coordinate is always free. Therefore, the points $x, y$ of the space $\widetilde{\mathcal{M}} \cong \mathbb{R}^{2} / \sim$ admit the disjoint neighborhoods defined, e.g., by the open balls of radius $1 / 3$ centered in $x$ and $y$, respectively. The balls are locally homeomorphic to the Euclidean plane, and therefore $\widetilde{\mathcal{M}}$ is a two-dimensional orbifold.

Lemma 1 gives a (partial) desingularization of the space $\mathcal{M}$. Indeed, we have seen that the group $S L(2, \mathbb{Z}) \times \mathbb{Z}$ acts in the plane $\left(\mathbb{A}_{\theta}, \operatorname{Ext}_{t}\left(\mathbb{A}_{\theta}, \mathbb{A}_{\theta}\right)\right)$ by the formula $(\theta, t) \mapsto\left(\frac{a \theta+b}{c \theta+d}, t+n\right)$, where $a d-b c=1$ and $a, b, c, d, n \in \mathbb{Z}$. However, the last formula does not specify the action on the parameter plane $(\theta, t)$ of the modular group $S L(2, \mathbb{Z})$ alone, since the function $n=n(a, b, c, d)$ is unknown. To find how the integer $n$ depends on the integers $a, b, c, d$, we would need a special construction which involves a correspondence (a covariant functor) between the complex and noncommutative tori. Such a construction will be given in the next paragraph and is encapsulated in the following lemma.

\footnotetext{
${ }^{1}$ That is, $f$ preserves the positive cone of $K$ and $\mathbb{R}: f\left(K^{+}\right)>0$.
} 
Lemma 2. There exists a homeomorphism $h: \widetilde{\mathcal{M}} \rightarrow \mathbb{H} / S L(2, \mathbb{Z})$, where $\mathbb{H}=$ $\{x+i y \in \mathbb{C} \mid y>0\}$ is the Lobachevsky plane endowed with a hyperbolic metric.

Proof of Lemma 2, Let $X$ be a topological surface of genus $g \geq 0$. The Teichmüller space $T_{g}$ of $X$ consists of the equivalence classes of the complex structures on $X$. The space $T_{g}$ is an open ball of the (real) dimension $6 g-6$ if $g \geq 2$ and $2 g$ if $g=0,1$. By $\operatorname{Mod} X$ we designate a group of the orientation-preserving diffeomorphisms of $X$ modulo the trivial ones. The points $S, S^{\prime} \in T_{g}$ are equivalent if there exists a conformal map $f \in \operatorname{Mod} X$ such that $S^{\prime}=f(S)$. The moduli of conformal equivalence is denoted by $\mathcal{M}_{g}=T_{g} / \operatorname{Mod} X$. The space $\mathcal{M}_{g}$ is a (classical) moduli space, whose definition dates back to Riemann.

Let $S \in T_{g}$ be a Riemann surface thought of as a point in the Teichmüller space, and let $H^{0}\left(S, \Omega^{\otimes 2}\right)$ be the space of the holomorphic quadratic forms on $S$. The fundamental theorem of Hubbard and Masur says that there exists a homeomorphism $h_{S}: H^{0}\left(S, \Omega^{\otimes 2}\right) \rightarrow T_{g}$ [6, p. 224]. The space $H^{0}\left(S, \Omega^{\otimes 2}\right)$ is a real vector space of dimension $6 g-6$, where $g \geq 2$. It has been shown in the above cited work that $H^{0}\left(S, \Omega^{\otimes 2}\right) \cong \operatorname{Hom}\left(H_{1}(\tilde{X}, \tilde{\Gamma})^{-} ; \mathbb{R}\right)$ defined by the formula

$$
\omega \longmapsto\left(\gamma \mapsto \operatorname{Im} \int_{\gamma} \omega\right)
$$

where $H_{1}(\tilde{X}, \tilde{\Gamma})^{-}$is the odd part in the homology of a double cover $\tilde{X}$ of $X$ ramified at the zeroes $\tilde{\Gamma}$ of the odd multiplicity of the quadratic form [6, p. 232]. (The symbols and formulas will simplify as we come to the complex torus - our principal case.) It has been proved that $H_{1}(\tilde{X}, \tilde{\Gamma})^{-} \cong \mathbb{Z}^{6 g-6}$.

Let $X=T^{2}$, i.e. $g=1$. In this case each quadratic differential form is the square of a holomorphic abelian form (a one-form), i.e. $H^{0}\left(S, \Omega^{\otimes 2}\right)=H^{0}(S, \Omega)$. Therefore $\tilde{X}=X=T^{2}, \tilde{\Gamma}=\emptyset$ and $H_{1}(\tilde{X}, \tilde{\Gamma})^{-}=H_{1}\left(T^{2}\right) \cong \mathbb{Z}^{2}$. In other words, one gets a homeomorphism $h_{S}: H o m\left(\mathbb{Z}^{2}, \mathbb{R}\right) \rightarrow T_{1}$. As we have seen earlier, Hom $\left(\mathbb{Z}^{2}, \mathbb{R}\right)=$ $\left\{t_{1} \mathbb{Z}+t_{2} \mathbb{Z} \mid t_{1}, t_{2} \in \mathbb{R}\right\}=\{t(\mathbb{Z}+\theta \mathbb{Z}) \mid \theta, t \in \mathbb{R}\}$, where $t=t_{1}, \theta=t_{2} / t_{1}$. On the other hand, the Teichmüller space $T_{1} \cong \mathbb{H}$, where $\mathbb{H}=\{\tau=x+i y \in \mathbb{C} \mid y>0\}$ is a (Lobachevsky) upper half-plane and $\tau$ is a modulus of the complex torus $\mathbb{C} /(\mathbb{Z}+\tau \mathbb{Z})$ [10, pp. 6-14]. Thus, we have a homeomorphism $h_{S}:\left(\mathbb{A}_{\theta}, E x t_{t}\left(\mathbb{A}_{\theta}, \mathbb{A}_{\theta}\right)\right) \rightarrow \mathbb{H}$.

Let us show that $h_{S}$ is equivariant in the first coordinate with respect to the action of $\operatorname{Mod}\left(T^{2}\right) \cong S L(2, \mathbb{Z})$; i.e., $\tau^{\prime} \equiv \tau \bmod S L(2, \mathbb{Z})$ if and only if $\theta^{\prime} \equiv$ $\theta \bmod S L(2, \mathbb{Z})$. Indeed, since Hom $\left(H_{1}\left(T^{2}\right) ; \mathbb{R}\right) \cong \mathbb{H}$ the modular group $S L(2, \mathbb{Z})$ acts on the right-hand side by the formula $\tau \mapsto(a \tau+b) /(c \tau+d)$ and on the left-hand side by a linear transformation $p_{1} \mapsto a p_{1}+b p_{2}, p_{2} \mapsto c p_{1}+d p_{2}$, where $p=\left(p_{1}, p_{2}\right) \in H_{1}\left(T^{2}\right)$ and $a d-b c=1$. The $f_{p}(t)=p_{1} t_{1}+p_{2} t_{2}$ will become $f_{p}\left(t^{\prime}\right)=t_{1}\left(a p_{1}+b p_{2}\right)+t_{2}\left(c p_{1}+d p_{2}\right)=p_{1} t_{1}^{\prime}+p_{2} t_{2}^{\prime}$, where $t_{1}^{\prime}=a t_{1}+c t_{2}$ and $t_{2}^{\prime}=b t_{1}+d t_{2}$. Therefore $\theta=t_{2} / t_{1}$ goes to $\theta^{\prime}=t_{2}^{\prime} / t_{1}^{\prime}=(b+d \theta) /(a+c \theta)$ and $\theta^{\prime} \equiv \theta \bmod S L(2, \mathbb{Z})$. (The 'only if' part of the statement is obtained likewise by an inversion of the formulas.)

Recall that the Lobachevsky plane $\mathbb{H}=\{x+i y \in \mathbb{C} \mid y>0\}$ carries a hyperbolic metric $d s=|d z| / y$ such that $S L(2, \mathbb{Z})$ acts on it by the isometries (linear-fractional transformations). The tesselation of $\mathbb{H}$ by the fundamental regions is shown in Figure 2. Let $\tau^{\prime}=\frac{a \tau+b}{c \tau+d}=T(\tau), \tau \in \mathbb{H}$. The number $n=n(a, b, c, d) \in \mathbb{Z}$ we shall call a height of the transformation $T \in S L(2, \mathbb{Z})$ if $n$ is equal to the number of intersections of the vertical segment $\operatorname{Im}\left(\tau^{\prime}-\tau\right)$ issued from $\tau$ with the lines of tiling $\mathbb{H} / S L(2, \mathbb{Z})$. (In other words, $n$ shows how many fundamental regions apart 


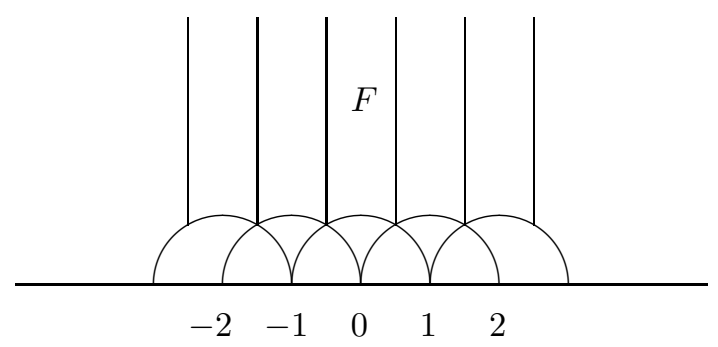

FiguRe 2. $\mathbb{H}$ and the fundamental region $F$.

from $\tau$ and $\tau^{\prime}$ are in the vertical direction.) We leave it to the reader to verify that the height $n$ does not depend on a particular choice of $\tau$ in the fundamental region or the fundamental region itself being a function of the transformation $T$ only.

Let us now define an action of the modular group on $\left(\mathbb{A}_{\theta}, E x t_{t}\left(\mathbb{A}_{\theta}, \mathbb{A}_{\theta}\right)\right)$. The action is given by the formula $(\theta, t) \mapsto\left(\frac{a \theta+b}{c \theta+d}, t+n\right)$, where $n=n(a, b, c, d)$ is the height of the transformation $T=T(a, b, c, d)$. Under the homeomorphism $h_{S}$, the tesselation of $\mathbb{H}$ maps into a tesselation of the plane $(\theta, t)$. As we have shown earlier, the action of the modular group $S L(2, \mathbb{Z})$ on $\mathbb{H}$ is equivariant with the action on $(\theta, t)$. On the other hand, it is known that $\mathbb{H} / S L(2, \mathbb{Z})$ is a punctured two-dimensional sphere [10, p. 15]. Lemma 2 and Theorem 1 follow.

\section{REMARKS}

Let $E_{\tau}=\mathbb{C} /(\mathbb{Z}+\tau \mathbb{Z})$ be a complex torus and $h_{S}\left(E_{\tau}\right)=\left(\mathbb{A}_{\theta}, \operatorname{Ext}_{t}\left(\mathbb{A}_{\theta}, \mathbb{A}_{\theta}\right)\right)$ its image under the homeomorphism $h_{S}$. Let us call the respective reals $\theta=\theta(\tau)$ and $t=t(\tau)$ a projective curvature and an area of the complex torus $E_{\tau}$. The projective curvature of the complex tori with a nontrivial group of endomorphisms (complex multiplication) is a quadratic irrationality. In the latter case, the noncommutative torus is said to have a real multiplication. The noncommutative tori with real multiplication can be used to construct the abelian extensions of the real quadratic number fields, as was suggested by Yu. Manin [7]. It seems challenging at this point to write a formula for the projective curvature and the area as a function of the complex modulus $\tau$. It is likely that the functions will be of the class $C^{0}$.

Problem 1. Find a formula (if any) for the functions $\theta(\tau)$ and $t(\tau)$.

\section{ACKNOWLEDGMENTS}

I am grateful to Michael Lamoureux for valuable help in the preparation of the manuscript. The referee's suggestions are kindly acknowledged and have been incorporated into the text. 


\section{REFERENCES}

[1] B. Blackadar, K-Theory for Operator Algebras, MSRI Publ. 5, Springer, 1986. MR859867 (88g:46082)

[2] E. Effros, Dimensions and $C^{*}$-Algebras, Conf. Board Math. Sci., vol. 46, AMS, 1981. MR623762 (84k:46042)

[3] E. Effros and C.-L. Shen, Approximately finite $C^{*}$-algebras and continued fractions, Indiana Univ. Math. J. 29 (1980), 191-204. MR563206 (81g:46076)

[4] K. R. Goodearl, Partially Ordered Abelian Groups with Interpolation, Mathematical Surveys and Monographs 20, AMS, 1986. MR845783 (88f:06013)

[5] D. Handelman, Extensions for AF $C^{*}$ algebras and dimension groups, Trans. Amer. Math. Soc. 271 (1982), 537-573 MR654850 (84e:46063)

[6] J. Hubbard and H. Masur, Quadratic differentials and foliations, Acta Math. 142 (1979), 221-274. MR523212 (80h:30047)

[7] Yu. I. Manin, Real multiplication and noncommutative geometry, in "The legacy of Niels Henrik Abel", 685-727, Springer, 2004. MR2077591(2006e:11077)

[8] M. Pimsner and D. Voiculescu, Imbedding the irrational rotation $C^{*}$-algebra into an AFalgebra, J. Operator Theory 4 (1980), 201-210. MR.595412 (82d:46086)

[9] M. A. Rieffel, $C^{*}$-algebras associated with irrational rotations, Pacific J. Math. 93 (1981), 415-429. MR623572 (83b:46087)

[10] J. H. Silverman, Advanced Topics in the Arithmetic of Elliptic Curves, Graduate Texts in Mathematics 151, Springer, 1994. MR1312368(96b:11074)

The Fields Institute for Mathematical Sciences, Toronto, Ontario, Canada

E-mail address: igor.v.nikolaev@gmail.com 